\title{
Efficacy and safety of ceftazidime-avibactam in the treatment of complicated intra-abdominal infections (CIAIs) and complicated urinary tract infections (CUTIs): A meta-analysis of randomized controlled trials
}

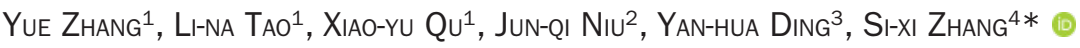 \\ ${ }^{1}$ MA.Sc, Department of Pharmacy, The First Hospital of Jilin University, Changchun, China \\ ${ }^{2} \mathrm{MD}$, PhD, Professor of the Department of Hepatology, The First Hospital of Jilin University, Changchun, China \\ ${ }^{3} \mathrm{MD}$, PhD, Professor of the Phase I Clinical Trial Unit, The First Hospital of Jilin University, Changchun, China \\ ${ }^{4} \mathrm{MD}$, PhD, Department of Pharmacy, The First Hospital of Jilin University, Changchun, China
}

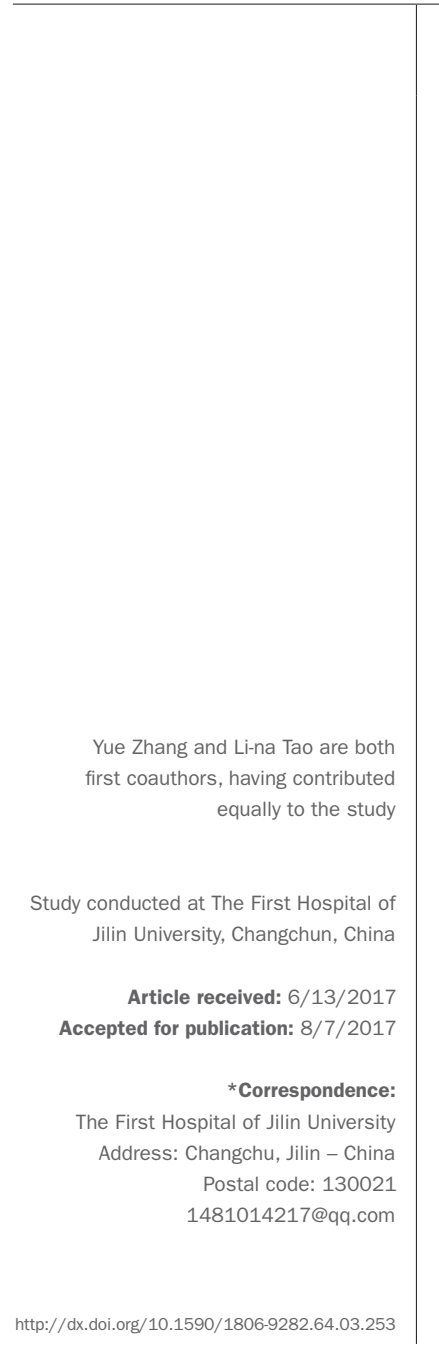

\section{SUMmARY}

\begin{abstract}
Objective: The aim of this study was to assess the efficacy and safety of ceftazidime-avibactam in the treatment of complicated intra-abdominal infections (CIAIs) and complicated urinary tract infections (CUTIs) with meta-analysis method. Method: We included six randomized clinical trials identified from Medline, Embase, Cochrane Library, "ISRCTN Register" and "ClinicalTrials.gov" which compared ceftazidime-avibactam with comparison group. The meta-analysis was performed using Review Manager software version 5.3.

Results: Ceftazidime-avibactam versus active comparisons demonstrated a statistically significant higher rate of microbiological response success on microbiological evaluable populations at the test-of-cure visit (95CI 1.10-2.38, $\mathrm{p}=0.02)$ and late-follow-up visit (95CI 1.09-2.23, $\mathrm{p}=0.02)$ for the treatment of CUTIs. Ceftazidime-avibactam versus active comparisons demonstrated a statistically significant higher rate of microbiological response success on EME populations at the test-of-cure visit (95CI 1.08-4.27, $\mathrm{p}=0.03$ ) and late-follow-up visit $(\mathrm{OR}=1.75$, 95CI 1.33-2.29, $\mathrm{p}<0.0001)$ for the treatment of CUTIs. Similar results were obtained at the late-follow-up visit $(\mathrm{OR}=1.58,95 \mathrm{CI} 1.26-1.97$, $\mathrm{p}<0.0001$ ) on microbiologically modified intent-to-treat (mMITT) populations for the treatment of CUTIs. We can find better eradication rates for E. coli and Klebsiella pneumoniae based on MMITT populations. In terms of AEs, SAEs and mortality, ceftazidime-avibactam had a safety and tolerability profile broadly similar to the comparison group.

Conclusion: This meta-analysis provides evidence of the efficacy of ceftazidime-avibactam as a potential alternative for the treatment of patients with CUTIs, and CIAIs.
\end{abstract}

Keywords: Meta-analysis. Ceftazidime. Azabicyclo Compounds. Urinary Tract Infections. Intra-abdominal Infections. Efficacy. Safety.

\section{INTRODUCTION}

The incidence of infection with antibiotic-resistant bacteria is steadily increasing in the world, and the treatment of drug-resistant bacterial infection has become a challenge. ${ }^{1}$ Complicated urinary tract infections (CUTIs) and complicated intra-abdominal infections (CIAIs) are problematic conditions frequently encountered by phy- sicians. CIAIs generally result from perforation or necrosis of the gastrointestinal tract and release of bacteria into the peritoneal and retroperitoneal space ${ }^{2}$ as well as postoperatively due to leaks or deep surgical wound infections. Among the Gram-negative pathogens associated with CIAIs, the most common are the Enterobacteriaceae, especially Escherichia coli (E.coli) and Klebsiella 
spp. ${ }^{3}$ CUTIs include emphysematous pyelonephritis, emphysematous pyelitis/cystitis, xanthogranulomatous pyelonephritis, renal/perirenal abscess and renal papillary necrosis. ${ }^{4}$ The bacterial epidemiology of CUTIs in this study is similar to that generally reported elsewhere in North America, Latin America and Europe. E. coli remains the most frequently isolated uropathogen, followed by Psendomonas aeruginosa (P. aeruginosa) and Proteus spp. ${ }^{5,6}$ E. coli is the predominant Gram-negative uropathogen. ${ }^{7}$ In the past three decades, $\beta$-lactam antibiotics including second-and third-generation cephalosporins, $\beta$-lactam $/ \beta$-lactamase inhibitor combinations and carbapenems have been widely used in clinical practice for the treatment of CIAIs and CUTIs because of their broad coverage of clinically important Gram-negative bacteria. ${ }^{8}$ Pathogens in these infections are often highly-resistant to standard antibiotics ${ }^{9,10}$ in some regions and countries. Thus, there is an urgent requirement for CIAI and CUTI therapies. ${ }^{11,12}$ Ceftazidime-avibactam has been shown to be effective in phase II clinical trials conducted in patients with CIAIs or CUTIs and has also been shown to be generally well tolerated, with a safety profile so far seen to be broadly similar to the established safety profile of ceftazidime. ${ }^{13-15}$ Ceftazidime-avibactam is an important new option for such cases in CIAIs (in combination with metronidazole) and CUTIs patients. Ceftazidime is a widely used expanded-spectrum anti-pseudomonal cephalosporin and avibactam with a novel non- $\beta$-lactam $\beta$-lactamase inhibitor. ${ }^{13,14}$ Ceftazidime-avibactam was recently granted accelerated approval by the FDA for the treatment of CUTIs and CIAIs combined with metronidazole in adult patients when treatment options are limited. ${ }^{16}$ Ceftazidime-avibactam is an antibacterial agent that consists of an existing third-generation cephalosporin combined with a novel $\beta$-lactamase inhibitor. An important advantage of ceftazidime-avibactam is that avibactam can expand the antibacterial activity of ceftazidime against Enterobacteriaceae and $P$. aeruginosa by inhibiting $\mathrm{AmpC}$, extended-spectrum $\beta$-lactamase, and carbapenemase producing strains. ${ }^{17}$ We conducted a meta-analysis of RCTs to clarify whether the use of ceftazidime-avibactam could be associated with improved outcomes in comparison with those achieved with other antibiotics for the treatment of infections, including CIAIs and CUTIs.

\section{Method}

\section{Search strategy and selection criteria}

To identify relevant randomized trials, we searched the literature through PubMed, Embase and Cochrane Library up to 30 June 2016 with the search strategies "ceftazidime-avibactam" or "ceftazidime/NXL104" and "randomized controlled trials" or "randomized" or "randomized." To identify relevant unpublished studies, we searched "ISRCTN Register" and "ClinicalTrials.gov" with the same search strategies up to 30 June 2016. In addition, we searched all references in the relevant articles and reviews for additional eligible studies.

Two reviewers (Zhang and Tao) searched and examined relevant studies independently. Individual RCTs on the efficacy and safety of ceftazidime-avibactam in comparison with other antibiotics for the treatment of patients with CIAIs and CUTIs were included for analysis. We excluded the following articles: experimental trials researched in animals, articles focusing on pharmacokinetic or pharmacodynamic variables and trials focusing on the in-vitro activity of ceftazidime-avibactam.

\section{Data extraction}

The following data were extracted from each study: year of publication, type of trial design, number of patients, antimicrobial agents and dosage used, treatment duration, time from treatment to test of cure, clinical and microbiological outcomes and adverse effects. Two reviewers (Zhang and Tao) independently extracted the relevant data. Disagreements were resolved by discussion with a third author $(\mathrm{Qu})$.

\section{Analyzed outcomes}

The efficacy outcomes of this meta-analysis were clinical treatment success (defined as "clinical cure"), clinical response and microbiological response, respectively assessed at the test-of-cure (TOC) visit, late-follow-up (LFU) visit and end-of-treatment (EOT) visit based on modified intent-to-treat (MITT) population, microbiologically modified intent-to-treat (mMITT) population, clinically evaluable (CE) population, microbiological evaluable (ME) population or extended microbiologically valuable (EME) population in each individual study and incidence of adverse events (AEs).

The MITT population consisted of patients who received at least one dose of the study drug and followed intention-to-treat principles. The MMITT population consisted of patients who met the clinical disease criteria and had $\geq 1$ pathogen identified at baseline. The CE population consisted of patients who met the disease definition and had received the scheduled study drug, with sufficient information to determine clinical outcome. The ME population was a subset of the CE population who also had microbiologically documented infections. 
The EME population was a subset of ME population. The safety population included all patients who received any IV study therapy.

Clinical cure was the disappearance of acute signs and symptoms related to infection with no requirement for further antibiotic therapy. Clinical response was defined as resolution of all or most pre-therapy signs or symptoms with no further requirement for antibiotics or surgery. Microbiological response was defined as eradication of baseline pathogen.

\section{Quality assessment}

The two reviewers (Zhang and Tao) independently extracted the relevant data. The methodological quality of the RCTs was evaluated using the Jadad scoring system ${ }^{18}$ on the basis of details of randomization, generation of random numbers, details of the double-blinding procedure, information on withdrawals and allocation concealment. One point was awarded for the specification of each criterion, with a maximum of 5. High-quality RCTs scored 3 or more points. The study quality assessment for unpublished trials could not be done because information on study design was not available from clinical trial registries.

\section{Statistical analysis}

The meta-analysis was done using Review Manager, version 5.3. We assessed heterogeneity with $\mathrm{Q}$ statistics generated from the $\chi^{2}$ test and inconsistency with $\mathrm{I}^{2}$ measure. ${ }^{19}$ Significant heterogeneity was judged with p-values less than 0.10 or $\mathrm{I}^{2}$ more than $50 \%$. The publication bias was assessed by using the funnel plot. We chose to use a Mantel-Haenszel fixed-effect model (FEM) for pooling odds ratio (OR) and 95\% confidence interval (CI) for all outcomes (including the MITT, mMITT, ME, EME and CE population) when heterogeneity was not significant. We chose to use a DerSimonian and Laird random-effects model (REM) when heterogeneity was obvious.

\section{Results}

\section{Study selection outcomes}

A total of 56 articles related to this study were retrieved from the literature and subjected to the selection process. Among the 56 potentially relevant articles, 35 were excluded because the studies described in these articles were non-RCTs or had no results available. Another 15 articles were excluded, as they described part of RCTs that had been already included in the meta-analysis. Finally, six randomized studies were included in the meta-analysis: five published trials and one unpublished trial (NCT01726023 as study 1).

\section{Study characteristics}

Table 1 shows the following characteristics of the included RCTs: study design, type of infection, number of patients (MITT, mMITT, CE, ME, EME), mean age, drug information and Jadad score. The meta-analysis was composed of six RCTs for CIAIs and CUTIs. All included studies were RCTs conducted between 2012 and 2016. All included trials were multinational studies. The total sample of the included trials was 3,259 subjects and all trials were conducted exclusively in populations aged 18-90 years. Most subjects in ceftazidime-avibactam groups (for CIAIs in combination with metronidazole) received ceftazidime-avibactam 2,000 mg of ceftazidime and 500 $\mathrm{mg}$ of avibactam as intravenous infusion every 8 hours, followed by metronidazole $(500 \mathrm{mg}$ as intravenous infusion every 8 hours) for CIAIs. ${ }^{13,20-22}$ That, except for one study, in which patients received different doses of ceftazidime-avibactam $(500 \mathrm{mg}$ of ceftazidime and $125 \mathrm{mg}$ of avibactam as intravenous infusion every 8 hours). ${ }^{14}$ The mean Jadad score of the five publication RCTs was 4.6 (rang 3-5) and four trials had a high score of 5.

\section{Clinical cure success for the treatment of CIAls}

Clinical cure success rate for the treatment of CIAIs in the mMITT sample was provided in three trials totaling 1,139 subjects. The ceftazidime-avibactam group was associated with lower rate of clinical cure success, but the difference was not significant at TOC visit $(\mathrm{p}=0.11$, Table $2)$, EOT visit ( $\mathrm{p}=0.44$, Table 2$)$ and LFU visit $(\mathrm{p}=0.23$, Table 2). Data on MITT patients for the treatment of CIAIs was provided only in one trial, and the clinical cure success rate of ceftazidime-avibactam group was also lower than that of the comparison group at TOC visit (1,043 patients, $\mathrm{OR}=0.84,95 \mathrm{CI} 0.60-1.17, \mathrm{p}=0.30$, Table 2), EOT visit (1,043 patients, $\mathrm{OR}=0.64$, 95CI 0.42-0.97, $\mathrm{p}=0.04$, Table 2 ) and LFU visit (1,043 patients, $\mathrm{OR}=0.94$, 95CI 0.68-1.30, $\mathrm{p}=0.71$, Table 2 ), but again the difference was not significant at TOC visit and LFU visit. The comparison therapy group was associated with significantly more patients achieving clinical cure treatment success at EOT visit in MITT patients. Clinical cure success rate for the treatment of CIAIs in the CE sample was provided in two trials. The ceftazidime-avibactam group was associated with lower rate of clinical cure success, but the difference was not significant at TOC visit ( $\mathrm{p}=0.66$, Table 2 ), EOT visit ( $\mathrm{p}=0.33$, Table 2$)$ and LFU visit ( $\mathrm{p}=0.81$, Table 2). Data on ME patients for the treatment of CIAIs were provided only in one trial. In both comparisons, ceftazidime-avibactam shows lower success rate than comparison group at TOC visit (212 patients, $\mathrm{OR}=0.74$, 


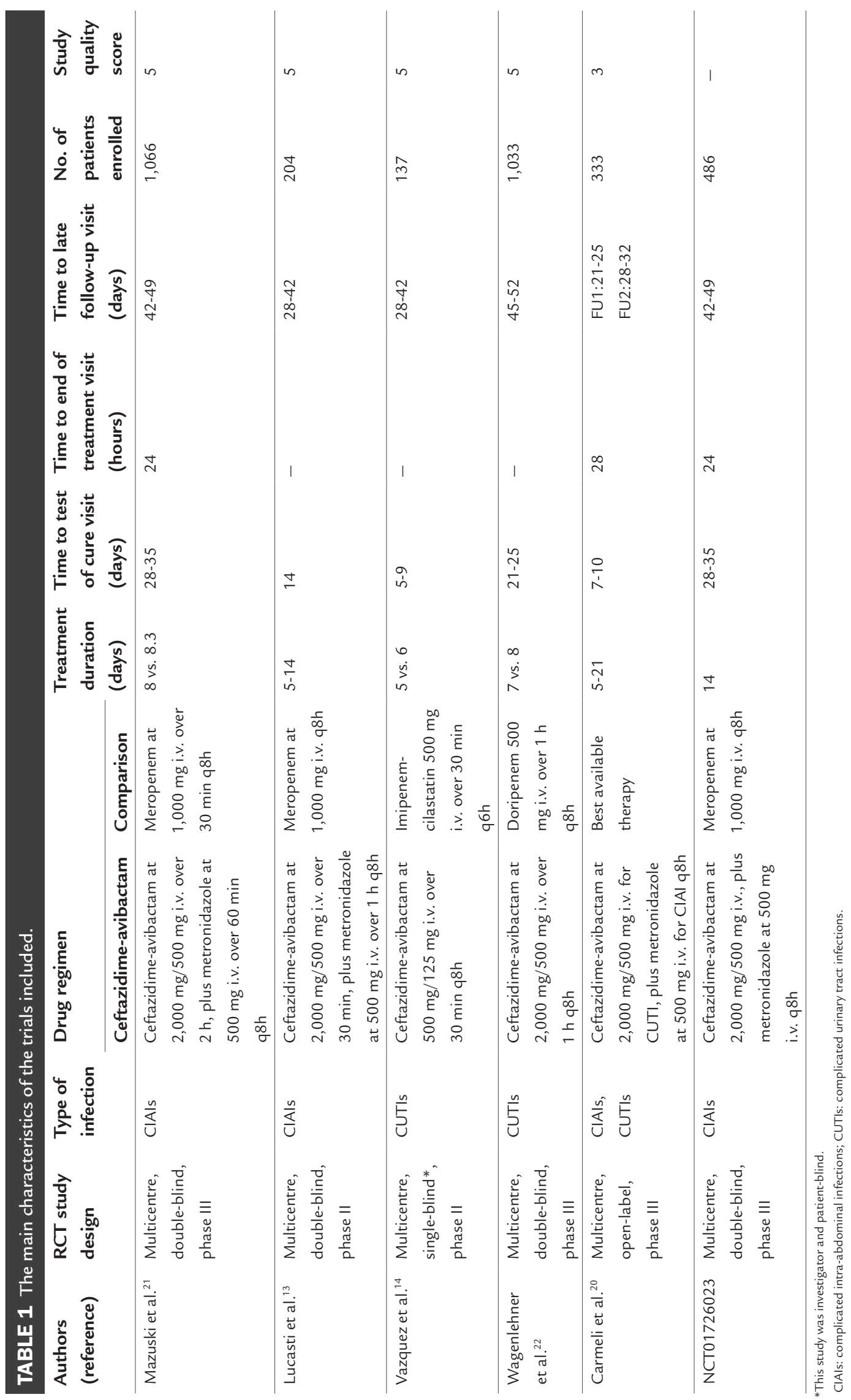


95CI 0.24-2.27, $\mathrm{p}=0.60$, Table 2 ) and LFU visit (202 patients, $\mathrm{OR}=0.76,95 \mathrm{CI} 0.25-2.35, \mathrm{p}=0.64$, Table 2), but at EOT visit shows higher success rate than comparison group (224 patients, OR = 1.52, 95CI 0.35-6.52, $\mathrm{p}=0.57$, Table 2) and the difference was not significant. Data on EME patients was provided in one trial, and the clinical cure success rate of the ceftazidime-avibactam group was also lower than that of the comparison group at TOC visit (219 patients, $\mathrm{OR}=0.71,95 \mathrm{CI} 0.23-2.17, \mathrm{p}=0.54$, Table 2), EOT visit (232 patients, $\mathrm{OR}=1.44$, 95CI 0.34-6.19, $\mathrm{p}=0.62$, Table 2 ) and LFU visit (209 patients, $\mathrm{OR}=0.73$, 95CI 0.24-2.24, $\mathrm{p}=0.58$, Table 2 ), but again the difference was not significant at TOC visit, EOT visit and LFU visit.

Clinical cure success for the treatment of CUTIs

Clinical cure success rate for the treatment of CUTIs on the mMITT sample was provided only in one trial. The ceftazi- dime-avibactam group was associated with lower rate of clinical cure success, but the difference was not significant at TOC visit, EOT visit or $L F U$ visit (for TOC visit, $\mathrm{p}=0.42$; for EOT visit, $\mathrm{p}=0.60$; for $\mathrm{LFU}$ visit, $\mathrm{p}=0.88$, Table 2 ). In our meta-analysis, the study of Carmeli et al. ${ }^{20}$ was split because in this study the LFU visit was divided into FU1 visit (21-25 days post-therapy) and FU2 visit (28-32 days post-therapy).

Microbiological response success for the treatment of CIAls Data on the microbiological response success for the treatment of CIAIs were provided in two of the included RCTs with mMITT and EME patients. For mMITT patients, in total, 127 (83.0\%) of the 153 patients in the ceftazidime-avibactam therapy group and 141 (86.5\%) of the 163 patients in the comparison therapy group achieved microbiological response success. The ceftazidime-avibactam therapy group failed to produce a significant difference in

TABLE 2 Effect of study/patient characteristics for the treatment of CIAls of clinical cure success and microbiological response success and for the treatment of CUTIs of clinical cure success.

\begin{tabular}{|c|c|c|c|c|c|c|}
\hline & Type of infection & $\begin{array}{l}\text { Treatment success } \\
\text { by population }\end{array}$ & Patients & Analysis model & Odds ratio $(95 \mathrm{Cl})$ & $\begin{array}{l}\text { Heterogeneity } \\
\left(\mathrm{I}^{2}, \mathrm{p} \text {-value }\right)\end{array}$ \\
\hline \multirow{15}{*}{$\begin{array}{l}\text { Clinical cure } \\
\text { success }\end{array}$} & \multirow[t]{15}{*}{ ClAls } & mMITT-TOC & 1,139 & FEM & $0.77(0.56-1.06)$ & $21 \%, 0.11$ \\
\hline & & mMITT-EOT & 1,139 & REM & $0.74(0.35-1.58)$ & $54 \%, 0.44$ \\
\hline & & mMITT-LFU & 1,139 & FEM & $0.82(0.60-1.13)$ & $21 \%, 0.23$ \\
\hline & & MITT-TOC & 1,043 & - & $0.84(0.60-1.17)$ &,- 0.30 \\
\hline & & MITT-EOT & 1,043 & - & $0.64(0.42-0.97)$ &,- 0.04 \\
\hline & & MITT-LFU & 1,043 & - & $0.94(0.68-1.30)$ &,- 0.71 \\
\hline & & CE-TOC & 1,187 & FEM & $0.91(0.59-1.41)$ & $0 \%, 0.66$ \\
\hline & & CE-EOT & 1,212 & FEM & $0.78(0.47-1.29)$ & $13 \%, 0.33$ \\
\hline & & CE-LFU & 1,173 & FEM & $0.95(0.64-1.43)$ & $0 \%, 0.81$ \\
\hline & & ME-TOC & 212 & - & $0.74(0.24-2.27)$ &,- 0.60 \\
\hline & & ME-EOT & 224 & - & $1.52(0.35-6.52)$ &,- 0.57 \\
\hline & & ME-LFU & 202 & - & $0.76(0.25-2.35)$ &,- 0.64 \\
\hline & & EME-TOC & 219 & - & $0.71(0.23-2.17)$ &,- 0.54 \\
\hline & & EME-EOT & 232 & - & $1.44(0.34-6.19)$ &,- 0.62 \\
\hline & & EME-LFU & 209 & - & $0.73(0.24-2.24)$ &,- 0.58 \\
\hline \multirow{9}{*}{$\begin{array}{l}\text { Microbiological } \\
\text { response success }\end{array}$} & \multirow[t]{9}{*}{ ClAls } & mMITT-TOC & 316 & REM & $1.11(0.23-5.29)$ & $61 \%, 0.89$ \\
\hline & & mMITT-EOT & 316 & REM & $1.68(0.16-18.03)$ & $73 \%, 0.67$ \\
\hline & & mMITT-LFU & 316 & REM & $1.12(0.25-5.08)$ & $59 \%, 0.88$ \\
\hline & & ME-TOC & 212 & - & $0.74(0.24-2.27)$ &,- 0.60 \\
\hline & & ME-EOT & 224 & - & $1.52(0.35-6.52)$ &,- 0.57 \\
\hline & & ME-LFU & 202 & - & $0.76(0.25-2.35)$ &,- 0.64 \\
\hline & & EME-TOC & 232 & - & $0.71(0.23-2.17)$ &,- 0.54 \\
\hline & & EME-EOT & 246 & - & $1.44(0.34-6.19)$ &,- 0.62 \\
\hline & & EME-LFU & 221 & - & $0.73(0.24-2.24)$ &,- 0.58 \\
\hline \multirow{3}{*}{$\begin{array}{l}\text { Clinical cure } \\
\text { success }\end{array}$} & \multirow[t]{3}{*}{ CUTIs } & mMITT-TOC & 281 & - & $0.68(0.27-1.72)$ &,- 0.42 \\
\hline & & mMITT-EOT & 281 & - & $0.52(0.05-5.82)$ &,- 0.60 \\
\hline & & mMITT-LFU & 562 & REM & $0.96(0.59-1.58)$ & $0 \%, 0.88$ \\
\hline
\end{tabular}

"-" shows that data in this study was provided only in one trial.

CIAIs: complicated intra-abdominal infections; CUTIs: complicated urinary tract infections; MITT: modified intent-to-treat; mMITT: microbiologically modified intent-to-treat; TOC: test-of-cure; LFU: late-follow-up; EOT: end-of-treatment; ME: microbiological evaluable; CE: clinically evaluable; EME: extended microbiologically valuable; FEM: fixed-effect model; REM: random-effects model. 
the number of patients achieving microbiological response sucess at $\mathrm{TOC}$ visit $(\mathrm{OR}=1.11,95 \mathrm{CI} 0.23-5.29$, Table 2$)$, EOT visit $(\mathrm{OR}=1.68,95 \mathrm{CI} 0.16-18.03$, Table 2$)$ and LFU visit $(\mathrm{OR}=1.12$, 95CI 0.25-5.08, Table 2). Similar results were confirmed in the EME analysis with patients with lower rate of microbiological response success in the ceftazidime-avibactam therapy group (at TOC visit, 232 patients, $\mathrm{OR}=0.71,95 \mathrm{CI} 0.23-2.17$; at EOT visit, 246 patients, $\mathrm{OR}=1.44,95 \mathrm{CI} 0.34-6.19$; at LFU visit, 221 patients, $\mathrm{OR}=0.73,95 \mathrm{CI} 0.24-2.24$, Table 2). Data on the microbiological response success for the treatment of CIAIs were provided in one of the included RCTs on ME patients. In both comparisons, ceftazidime-avibactam shows lower success rate than comparison group at TOC visit $(\mathrm{p}=0.60$, Table 2 ) and LFU visit ( $\mathrm{p}=0.64$, Table 2 ), but at EOT visit shows higher success rate than comparison group $(\mathrm{p}=0.57$, Table 2) and the difference was not significant.

\section{Microbiological response success for the treatment of CUTIs}

Data on the microbiological response success for the treatment of CUTIs were provided in two of the included RCTs with mMITT patients. The ceftazidime-avibactam group was associated with higher rate of microbiological response success, but the difference was not significant at TOC visit and EOT visit (for TOC visit, $\mathrm{p}=0.05$, Figure $1 \mathrm{~A}$; for EOT visit, $\mathrm{p}=0.87$, Figure 1B). In total, $470(69.0 \%)$ of the 681 patients in the ceftazidime-avibactam therapy group and 405 (58.6\%) of the 691 patients in the comparison therapy group achieved microbiological response success at LFU visit. The ceftazidime-avibactam therapy group was associated with significantly more patients achieving microbiological response success at LFU visit $(\mathrm{OR}=1.58,95 \mathrm{CI} 1.26-1.97, \mathrm{p}<0.0001$, Figure 1C). Data on the microbiological response success for the treatment of CUTIs were provided in one of the included RCTs on MITT patients. In both comparisons, ceftazidime-avibactam shows higher success rate than comparison group at TOC visit (95 patients, $\mathrm{OR}=1.20,95 \mathrm{CI} 0.51-2.80, \mathrm{p}=0.67$, data not shown in the figure) and LFU visit (95 patients, $\mathrm{OR}=1.13,95 \mathrm{CI} 0.51-2.53, \mathrm{p}=0.77$, data not shown in the figure), but at EOT visit shows lower success rate than comparison group (95 patients, $\mathrm{OR}=0.59,95 \mathrm{CI} 0.16-2.25$, $\mathrm{p}=0.44$, data not shown in the figure) and the difference was not significant. Data on the microbiological response success for the treatment of CUTIs were provided in two of the included RCTs on ME patients. In all, 260 (83.1\%) of the 313 patients in the ceftazidime-avibactam therapy group and 250 (75.1\%) of the 333 patients in the comparison therapy group achieved microbiological response success at TOC visit. The ceftazidime-avibactam therapy group was associated with significantly more patients achieving microbiological response success at TOC visit $(\mathrm{OR}=1.61,95 \mathrm{CI} 1.10-2.38, \mathrm{p}=0.02$, Figure 1D). In all, 197 (72.7\%) of the 271 patients in the ceftazidime-avibactam therapy group and 184 (63.0\%) of the 292 patients in the comparison therapy group achieved microbiological response success at LFU visit. The ceftazidime-avibactam therapy group was associated with significantly more patients achieving microbiological response success at $\mathrm{LFU}$ visit $(\mathrm{OR}=1.56,95 \mathrm{CI} 1.09-2.23, \mathrm{p}=0.02$, Figure $1 \mathrm{~F})$. The ceftazidime-avibactam group was associated with a lower rate of microbiological response success and the difference was no significant at EOT visit $(\mathrm{p}=0.85$, Figure 1E). The treatment success of two RCTs was based on EME populations. There was no significant difference in treatment success at EOT visit between patients treated with ceftazidime-avibactam and those treated with comparisons $(\mathrm{p}=0.97$, Figure $1 \mathrm{H})$. However, in EME populations, the success of ceftazidime-avibactam treatment in the CUTIs subgroup was significantly higher than that in the comparison groups at the TOC visit and LFU visit (for TOC visit, 858 patients, $\mathrm{OR}=2.15$, 95CI 1.08-4.27, $\mathrm{p}=0.03$, Figure $1 \mathrm{G}$; for the LFU visit, 1,001 patients, $\mathrm{OR}=$ $1.75,95 \mathrm{CI} 1.33-2.29, \mathrm{p}<0.0001$, Figure 1I). In our metaanalysis, this part of the study of Carmeli et al. ${ }^{20}$ was split because in this study the LFU visit was divided into FU1 visit (21-25 days post-therapy) and FU2 visit (28-32 days post-therapy) for mMITT patients and EME patients.

Microbiological response success in the treatment of $\mathrm{mMITT}$ populations infected with E. coli, Klebsiella pneumoniae, and $P$. aeruginosa

Four RCTs included in the meta-analysis reported data on mMITT patients. In our meta-analysis, the study of Carmeli et al. ${ }^{20}$ was split according to the treatment of CUTIs and CIAIs. The total microbiological treatment success for the ceftazidime-avibactam group was numerically higher than that for the comparison group in the MMITT population at the TOC visit with significant difference (FEM, $\mathrm{OR}=1.36,95 \mathrm{CI} 1.01-1.82, \mathrm{p}=0.04$, data not shown in the figure). More specifically, treatment with ceftazidime-avibactam was associated with numerically higher eradication rates for E. coli and Klebsiella pneumoniae (for E. coli, REM, $\mathrm{OR}=1.11$, 95CI 0.51-2.43, $\mathrm{p}=0.79$; for Klebsiella pneumoniae, $\mathrm{FEM}, \mathrm{OR}=1.56,95 \mathrm{CI} 0.97-2.49, \mathrm{p}=0.06$, data not shown in the figure). Treatment with ceftazidime-avibactam was associated with numerically lower eradication rates for $P$. aeruginosa (FEM, OR $=0.71,95 \mathrm{CI} 0.31-1.64, \mathrm{p}=0.43$, data not shown in the figure). However, there were no significant differences in eradication for all these species. 
Study or subgroup

Experimental Control

Odds ratio

Odds ratio

Events Total Events Total Weight $\mathrm{M}-\mathrm{H}$, random, $95 \mathrm{Cl}$

$\mathrm{M}-\mathrm{H}$, random, $95 \mathrm{Cl}$

A mMITT-TOC

Carmeli et al. ${ }^{20}$

$\begin{array}{llllll}118 & 144 & 88 & 137 & 42.8 \% & 2.53[1.46,4.38]\end{array}$

$\begin{array}{lllllll}\text { Wagenlehner et al. }^{22} & 299 & 393 & 291 & 417 & 57.2 \% & 1.38[1.01,1.88]\end{array}$

Subtotal $(95 \mathrm{Cl})$

$\begin{array}{llll}537 & 554 & 100.0 \% & 1.79[0.99,3.22]\end{array}$

Total events

417

379

Heterogeneity: $\mathrm{Tau}^{2}=0.13 ; \mathrm{Chi}^{2}=3.54, \mathrm{df}=1(\mathrm{p}=0.06) ; \mathrm{I}^{2}=72 \%$

Test for overall effect: $Z=1.93(p=0.05)$

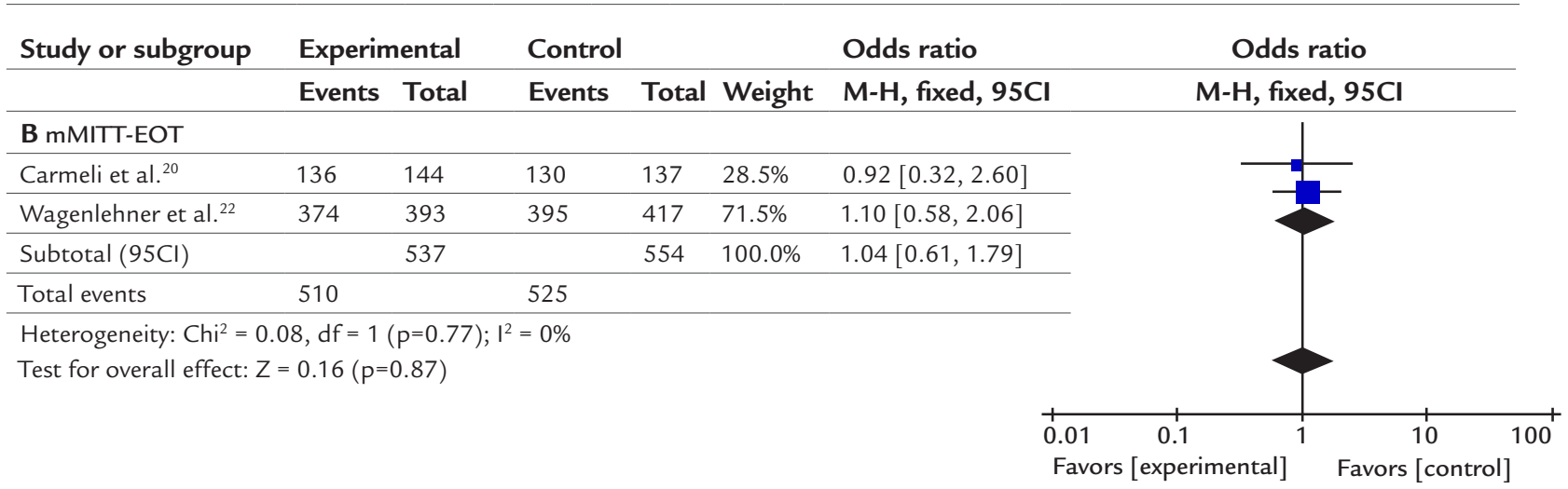

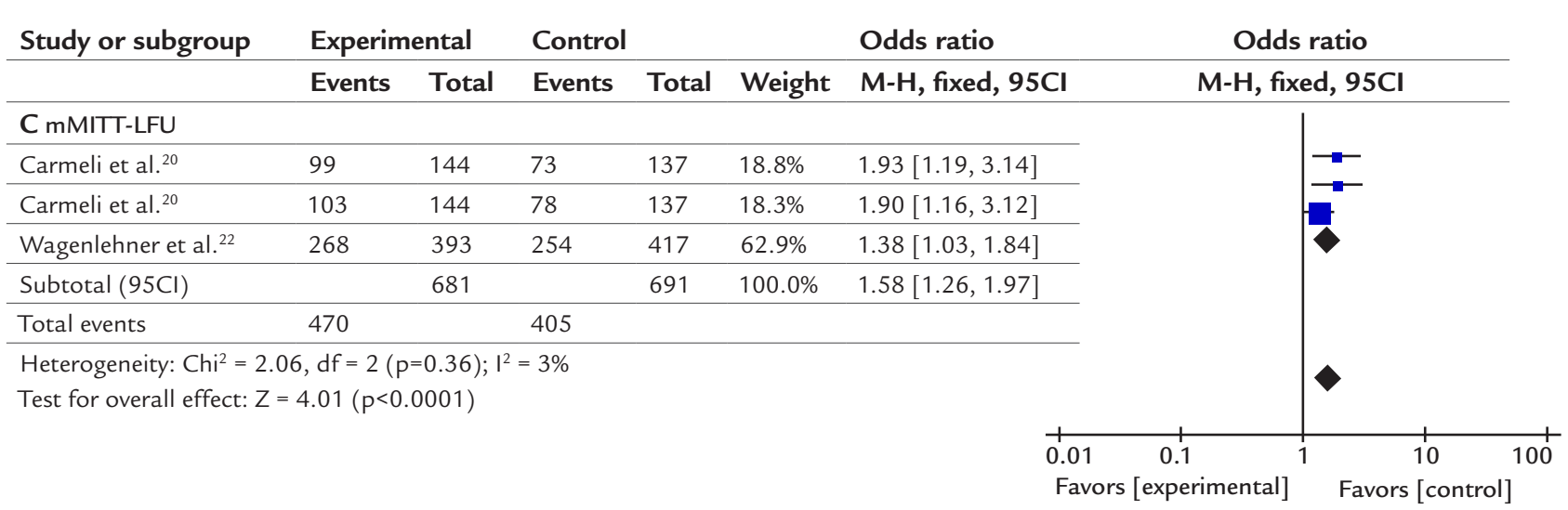

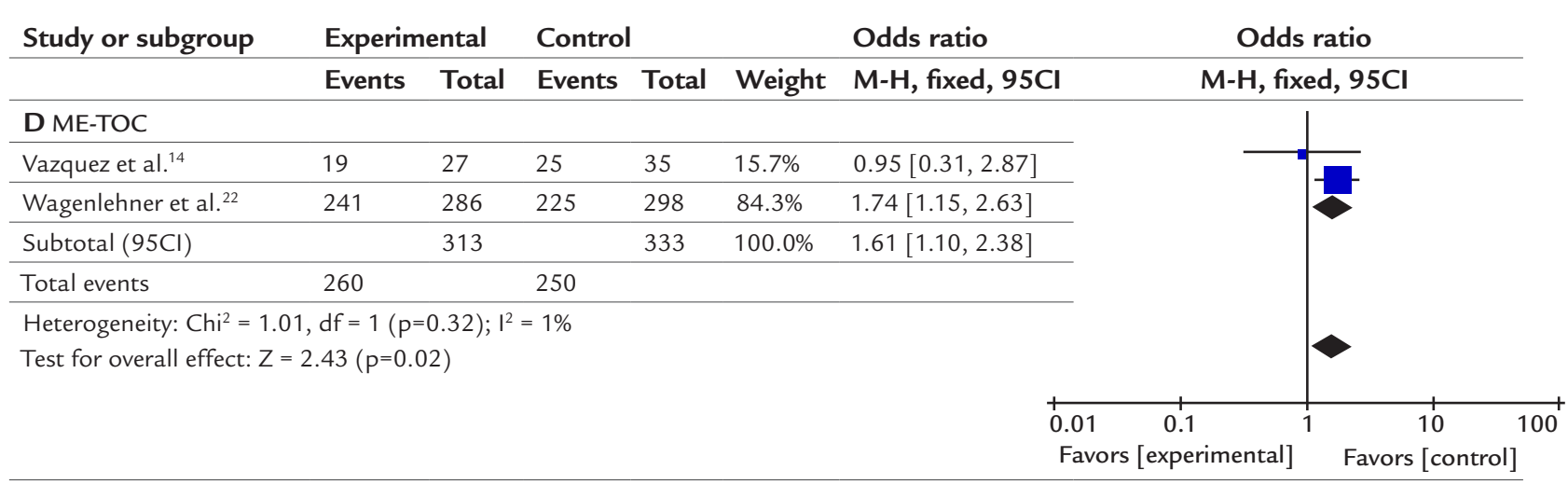




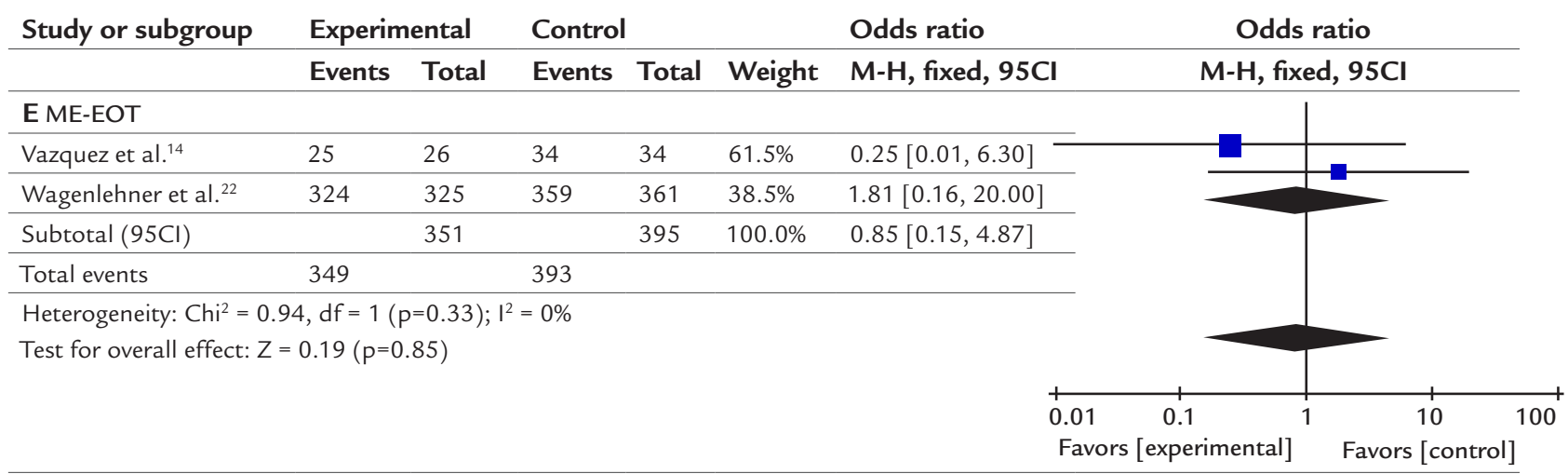

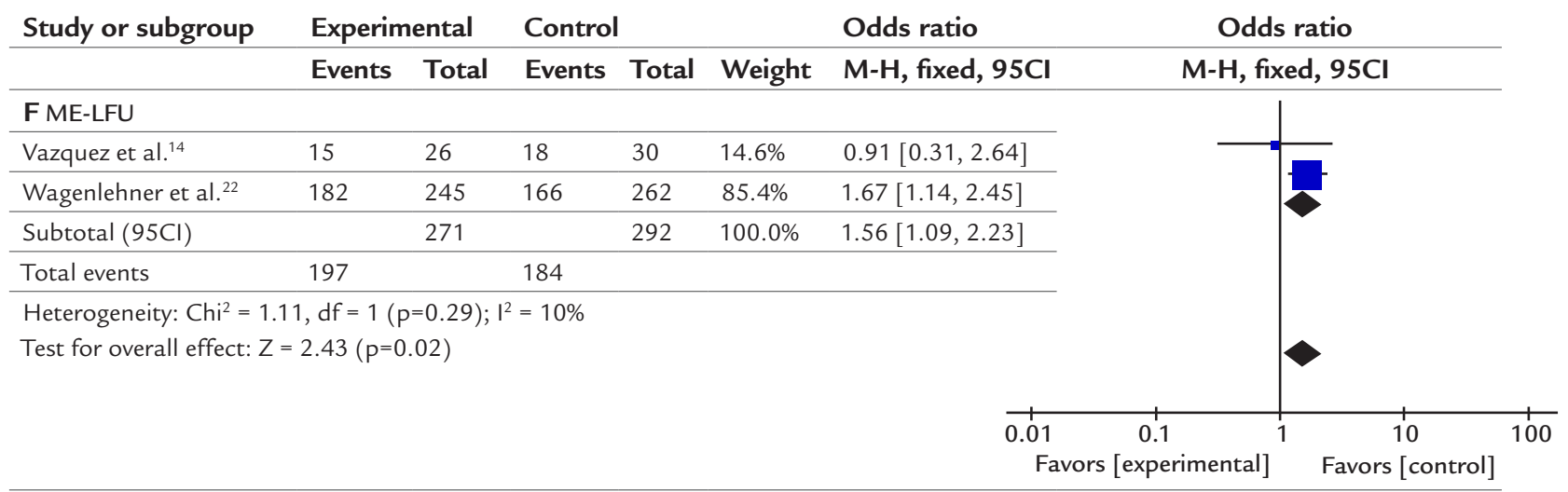

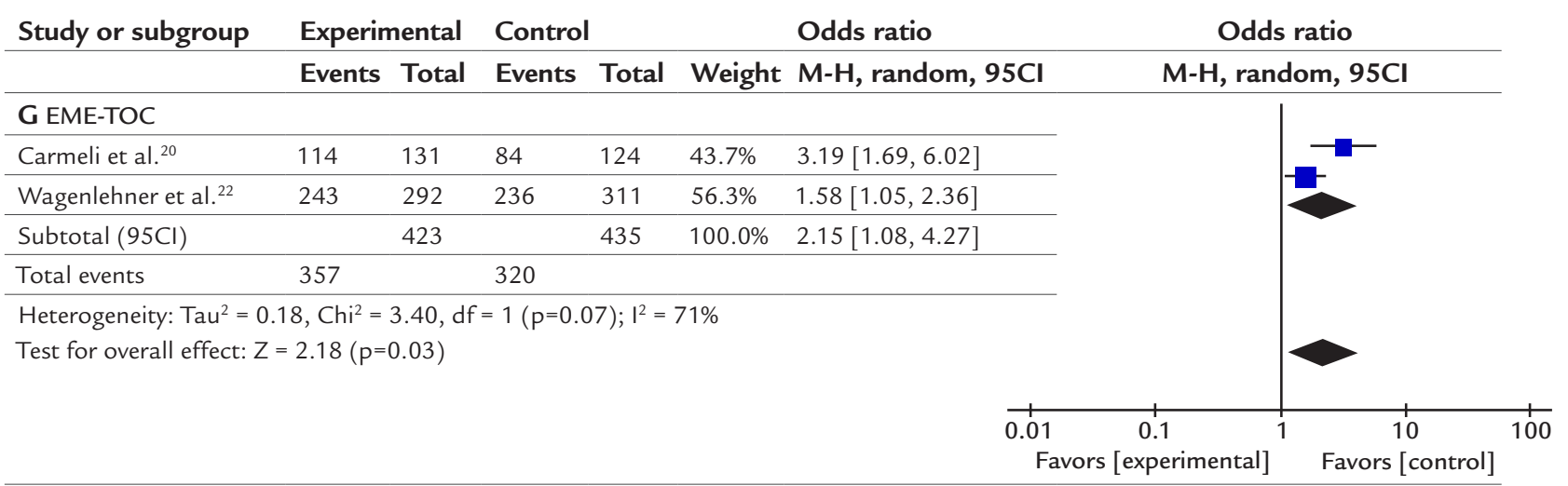

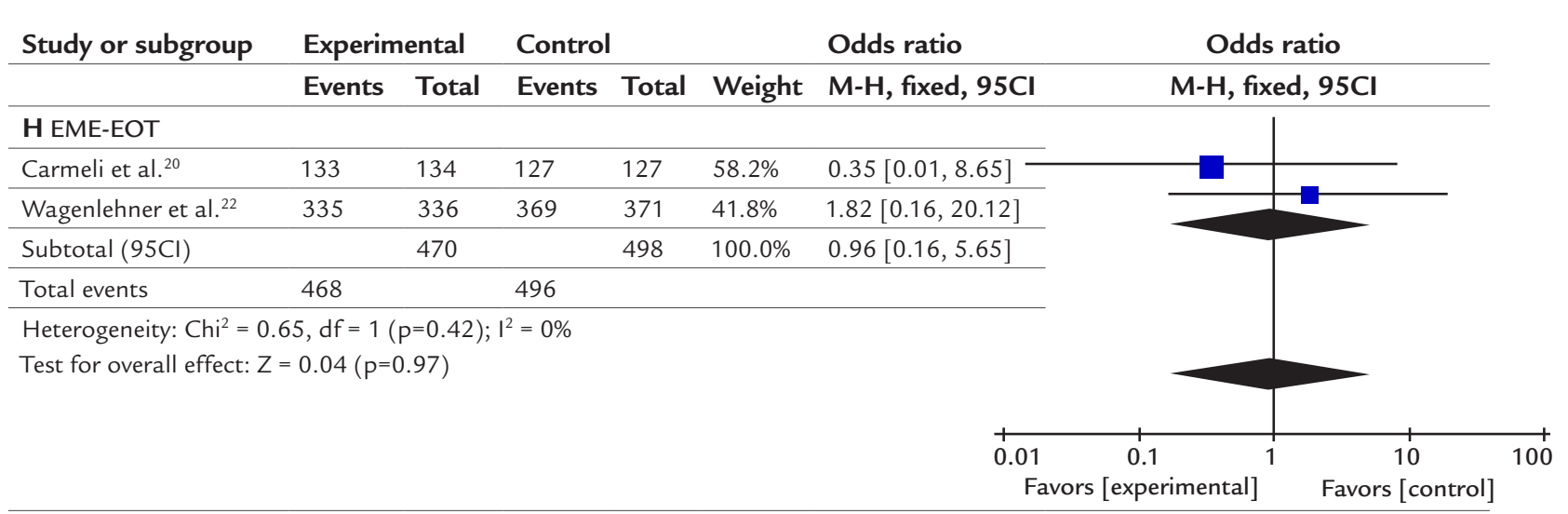




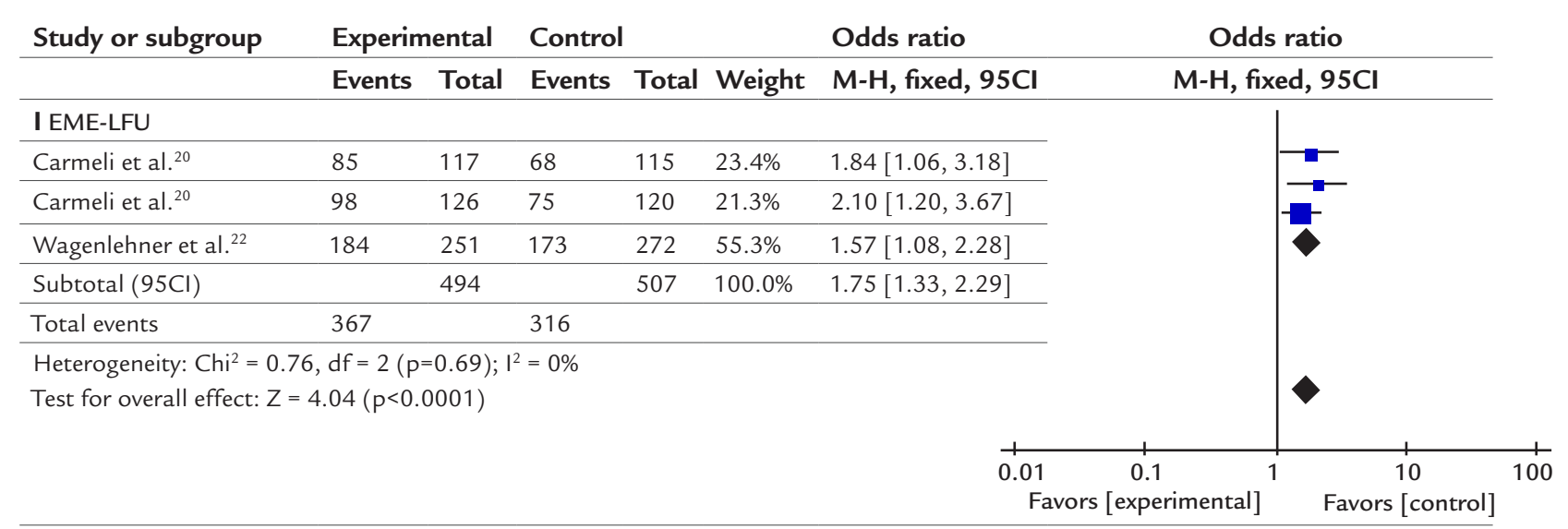

FIGURE 1 Meta-analysis of microbiological response success for the treatment of CUTIs based on mMITT populations, ME populations and EME populations: (A) microbiological response success at test-of-cure visit on mMITT populations; (B) microbiological response success at end-of-treatment visit on mMITT populations; (C) microbiological response success at late-follow-up visit on mMITT populations; (D) microbiological response success at test-of-cure visit on ME populations; (E) microbiological response success at end-of-treatment visit on ME populations; (F) microbiological response success at late-follow-up visit on ME populations; (G) microbiological response success at test-of-cure visit on EME populations; $(\mathrm{H})$ microbiological response success at end-of-treatment visit on EME populations; (I) microbiological response success at late-follow-up visit on EME populations. Vertical line indicates no difference between linezolid and vancomycin. The size of each square denotes the proportion of information given by each trial. Cl: confidence interval.

\section{Microbiological response success in the treatment of ME populations infected with E. coli, Klebsiella pneumoniae, and $P$. aeruginosa}

Three RCTs included in the meta-analysis reported data on ME patients. The total microbiological treatment success for the ceftazidime-avibactam group was numerically lower than that for the comparison group in the ME population at the TOC visit, but there was no significant difference (144 patients, $\mathrm{OR}=0.73,95 \mathrm{CI} 0.21-2.50, \mathrm{p}=0.61$, data not shown in the figure). More specifically, treatment with ceftazidime-avibactam was associated with numerically lower eradication rates for E. coli, Klebsiella pneumoniae, and P. aeruginosa (for E. coli, 309 strains, $\mathrm{OR}=0.88,95 \mathrm{CI}$ 0.42-1.87, $\mathrm{p}=0.74$; for Klebsiella pneumoniae, 68 strains, OR $=0.75,95 \mathrm{CI} 0.04-12.70, \mathrm{p}=0.84$; for $P$. aeruginosa, 42 strains, $\mathrm{OR}=1.86,95 \mathrm{CI} 0.15-23.00, \mathrm{p}=0.63$, data not shown in the figure). However, there were no significant differences in eradication for all these species.

\section{Adverse effects}

Data on AEs possibly or probably related to the study medications were reported in all of the included trials. According to the data in the meta-analysis, ceftazidime-avibactam was numerically higher than comparisons on incidence of AEs, but the difference was not significant $(3,180$ subjects, $\mathrm{OR}=1.09,95 \mathrm{CI} 0.94-1.25, \mathrm{p}=0.26$, data not shown in the figure). There was no significant difference in the proportions of patients who developed serious adverse events
(SAEs) in the ceftazidime-avibactam groups and comparison groups ( $\mathrm{six}$ RCTs, 3,180 subjects, OR = 1.14, 95CI 0.84$1.54, \mathrm{p}=0.40$, data not shown in the figure). In our metaanalysis, the study of Carmeli et al. ${ }^{20}$ was split because the treatment of CIAIs and CUTIs were both used in this study.

\section{Mortality}

All-cause mortality and mortality possibly related to the study drug during the study period were available in four of the six included trials. Although numerically higher mortality was found in the ceftazidime-avibactam groups, there was no significant difference in mortality between the ceftazidime-avibactam and comparison groups $(2,029$ patients, FEM, OR = 1.36, 95CI 0.70-2.65, $\mathrm{p}=0.37$, data not shown in the figure). In our meta-analysis, the study of Carmeli et al. ${ }^{20}$ was split because the treatment of CIAIs and CUTIs were both used in this study.

\section{Discussion}

Our study is the first systematic review with meta-analysis comparing the efficacy and safety of ceftazidime-avibactam with comparison for CIAIs and CUTIs. Therefore, it provides valuable information for clinicians and represents an important addition to the ceftazidime-avibactam trial program, providing supporting data for the treatment of CIAIs and CUTIs.

The results of our meta-analysis suggest that ceftazidime-avibactam is as effective as comparison antibiotics for 
the treatment of patients with CUTIs and CIAIs. Six RCTs, five published and one unpublished (four double blinded, one open labeled), met the inclusion criteria of our metaanalysis. Our findings suggest that ceftazidime-avibactam shows comparable efficacy in clinical cure and microbiological response compared with meropenem and best available therapy for CIAIs on mMITT, ME and EME populations at the TOC visit, EOT visit and LFU visit. Similarly, there was no significant difference in the numbers of clinical cure success between patients treated with ceftazidime-avibactam and the imipenem-cilastatin, doripenem or best available therapy for CUTIs based on MMITT populations at the TOC visit, EOT visit and LFU visit in these RCTs. Ceftazidime-avibactam versus active comparison drugs demonstrated a statistically significant higher rate of microbiological response success in ME and EME populations at the TOC visit and LFU visit for the treatment of CUTIs. Similar results are presented at the LFU visit on MMITT populations for the treatment of CUTIs. E. coli remains the most frequently isolated uropathogen, followed by $P$. aeruginosa and Proteus spp. Although ceftazidime-avibactam therapy showed no significant difference in eradiation rate from that of the comparison groups for almost all types of pathogens, we can find better eradication rates for E. coli and Klebsiella pneumoniae based on mMITT populations. Based on statistics of these three pathogens, we found no significant difference in eradication rate. One possible reason for that is the relatively small number of patients included. If more RCTs were included in the meta-analysis, the bacterial eradication rate would be more convincing.

Our meta-analysis revealed that there was no significant difference in the numbers of AEs, SAEs and mortality between patients treated with ceftazidime-avibactam and the comparison drugs. AEs occur predominantly in the gastrointestinal tract (including diarrhea, nausea, vomiting), nervous system (including headache, dizziness) and liver (including alanine aminotransferase increased, aspartate aminotransferase increased), and were also confirmed to be the adverse drug reactions most often reported in both comparison groups and clinically infected patients in many other studies of ceftazidime-avibactam treatment. Considering the known safety profile for metronidazole (for the treatment of CIAIs), no new safety signals for ceftazidime-avibactam were identified, and the overall safety profile was similar to that reported for ceftazidime alone and the cephalosporin class. Most AEs were mild or moderate in both groups, with low incidences of discontinuations or death due to AEs and few SAEs.

We attempted to apply best practices in this systematic review. Its strengths are: (1) as far as we know, this is the first systematic review with meta-analysis comparing the efficacy and safety of ceftazidime-avibactam with comparison for CIAIs and CUTIs; (2) in the present meta-analysis, we considered each infection separately (CUTIs and CIAIs) thereby reducing clinical heterogeneity at the design level; (3) in the present meta-analysis, we considered each populations separately (mMITT populations, MITT populations, CE populations, ME populations and EME populations) thereby reducing clinical heterogeneity at the design level; (4) in the present meta-analysis, we considered clinical cure success and microbiological response success thereby reducing clinical heterogeneity at the design level.

The findings of the present study must be viewed in the context of potential limitations. First, in most of the included trials, the comparison antibiotic was not the first-line recommended antibiotic for the assessed indication. Second, all RCTs included in our review were industry sponsored, involving authors related to the drug company. Such trials are more likely to report positive outcomes than trials funded by other sources. ${ }^{23}$ Third, the meta-analysis is based on a relatively small number of RCTs and we acknowledge that using a limited number of studies raises the possibility of a second-order sampling error. ${ }^{24}$ Fourth, there is heterogeneity in some of the relevant aspects (for example: comparative drugs included and different kinds of pathogens). Given this uncertainty resulting from clinical heterogeneity, subgroup analysis should be performed on different patients and comparative drugs about treatment success.

\section{Conclusion}

In conclusion, ceftazidime-avibactam as a potential alternative to carbapenems for treating CUTIs and CIAIs was effective and comparable to those of ceftazidime, metronidazole, meropenem and best available therapy and so on. It can play an important role in patients with Gram-negative pathogens resistant to ceftazidime and similar to its own efficacy against ceftazidime-susceptible infections. However, to obtain more definite conclusions, further investigation on ceftazidime-avibactam treatment is warranted.

\section{ACKNOWLEDGMENTS}

All authors revised the final manuscript and confirmed that it would not be published anywhere else.

\section{FUNDING}

This work was supported by the international standard for clinical trial technology platform construction of liver diseases under grant number 2014ZX09303303. 


\section{Ethical apProval}

Not required.

\section{RefERences}

1. Souli M, Galani I, Giamarellou H. Emergence of extensively drug-resistant and pandrug-resistant Gram-negative bacilli in Europe. Euro Surveill. 2008;13(47). Pii : 19045.

2. Sartelli M, Viale P, Catena F, Ansaloni L, Moore E, Malangoni M, et al. 2013 WSES guidelines for management of intra-abdominal infections. World $\mathrm{J}$ Emerg Surg. 2013;8(1):3.

3. Solomkin JS, Mazuski JE, Bradley JS, Rodvold KA, Goldstein EJ, Baron EJ, et al. Diagnosis and management of complicated intra-abdominal infection in adults and children: guidelines by the Surgical Infection Society and the Infectious Diseases Society of America. Clin Infect Dis. 2010;50(2):133-64.

4. Mnif MF, Kamoun M, Kacem FH, Bouaziz Z, Charfi N, Mnif F, et al. Complicated urinary tract infections associated with diabetes mellitus: pathogenesis, diagnosis and management. Indian J Endocrinol Metab. 2013;17(3):442-5.

5. Foxman B. The epidemiology of urinary tract infections. Nat Rev Urol. 2010;7(12):653-60.

6. Wagenlehner FM, Cek M, Naber KG, Kiyota H, Bjerklund-Johansen TE. Epidemiology, treatment and prevention of healthcare-associated urinary tract infections. World J Urol. 2012;30(1):59-67.

7. Castanheira M, Farrell SE, Krause KM, Jones RN, Sader HS. Contemporary diversity $\beta$-lactamase among Enterobacteriaceae in the nine U.S. census regions and ceftazidime-avibactam activity tested against isolates producing the most prevalent $\beta$-lactamase groups. Antimicrob Agents Chemother. 2014;58(2):833-8.

8. Mawal Y, Critchley IA, Riccobene TA, Talley AK. Ceftazidime-avibactam for the treatment of complicated urinary tract infections and complicated intraabdominal infections. Expert Rev Clin Pharmacol. 2015;8(6):691-707.

9. Nordmann P, Poirel L, Walsh TR, Livermore DM. The emerging NDM carbapenemases. Trends Microbiol. 2011;19(12):588-95.

10. Perez F, Van Duin D. Carbapenem-resistant Enterobacteriaceae: a menace to our most vulnerable patients. Cleve Clin J Med. 2013;80(4):225-33.

11. Drawz SM, Bonomo RA. Three decades of beta-lactamase inhibitors. Clin Microbiol Rev. 2010;23(1):160-201.

12. Kanj SS, Kanafani ZA. Current concepts in antimicrobial therapy against resistant gram-negative organisms: extended-spectrum beta-lactamase-producing Enterobacteriaceae, carbapenem-resistant Enterobacteriaceae, and multidrugresistant Pseudomonas aeruginosa. Mayo Clin Proc. 2011;86(3):250-9.

13. Lucasti C, Popescu I, Ramesh MK, Lipka J, Sable C. Comparative study of the efficacy and safety of ceftazidime/avibactam plus metronidazole versus meropenem in the treatment of complicated intra-abdominal infections in hospitalized adults: results of a randomized, double-blind, Phase II trial. J Antimicrob Chemother. 2013;68(5):1183-92.

14. Vazquez JA, González Patzán LD, Stricklin D, Duttaroy DD, Kreidly Z, Lipka $\mathrm{J}$, et al. Efficacy and safety of ceftazidime-avibactam versus imipenemcilastatin in the treatment of complicated urinary tract infections, including acute pyelonephritis, in hospitalized adults: results of a prospective, investigator-blinded, randomized study. Curr Med Res Opin. 2012;28(12):1921-31.

15. Merdjan H, Rangaraju M, Tarral A. Safety and pharmacokinetics of single and multiple ascending doses of avibactam alone and in combination with ceftazidime in healthy male volunteers: results of two randomized, placebocontrolled studies. Clin Drug Investig. 2015;35(5):307-17.

16. Sharma R, Park TE, Moy S. Ceftazidime-avibactam: a novel cephalosporin/ $\beta$-lactamase inhibitor combination for the treatment of resistant gramnegative organisms. Clin Ther. 2016;38(3):431-44.

17. Endimiani A, Choudhary Y, Bonomo RA. In vitro activity of NXL104 in combination with beta-lactams against Klebsiella pneumoniae isolates producing KPC carbapenemases. Antimicrob Agents Chemother. 2009;53(8):3599-601.

18. Adie S1, Harris IA, Naylor JM, Mittal R. Assessing the quality of report of randomized clinical trials: is blinding necessary. Control Clin Trials. 1996;17:1-12.

19. Higgins JP, Thompson SG, Deeks JJ, Altman DG. Measuring inconsistency in meta-analyses. BMJ. 2003;327(7414):557-60.

20. Carmeli Y, Armstrong J, Laud PJ, Newell P, Stone G, Wardman A, et alhttps:// www.ncbi.nlm.nih.gov/pubmed/?term=Gasink LB\%5BAuthor\%5D\&cauth or=true\&cauthor_uid=27107460. . Ceftazidime-avibactam or best available therapy in patients with ceftazidime-resistant Enterobacteriaceae and Pseudomonas aeruginosa complicated urinary tract infections or complicated intra-abdominal infections (REPRISE): a randomised, pathogen-directed, phase 3 study. Lancet Infect Dis. 2016;16(6):661-73.

21. Mazuski JE, Gasink LB, Armstrong J, Broadhurst H, Stone GG, Rank D, et al. Efficacy and safety of ceftazidime-avibactam plus metronidazole versus meropenem in the treatment of complicated intra-abdominal infection: results from a randomized, controlled, double-blind, phase 3 program. Clin Infect Dis. 2016;62(11):1380-9.

22. Wagenlehner FM, Sobel JD, Newell P, Armstrong J, Huang X, Stone GG, et al. Ceftazidime-avibactam versus doripenem for the treatment of complicated urinary tract infections, including acute pyelonephritis: RECAPTURE, a phase 3 randomized trial program. Clin Infect Dis 2016;63(6):754-62

23. Bourgeois FT, Murthy S, Mandl KD. Outcome reporting a mong drug tri als registered in ClinicalTrials.gov. Ann Intern Med. 2010;153(3):158-66.

24. Higgins J, Thompson S, Deeks J, Altman D. Statistical heterogeneity in systematic reviews of clinical trials: a critical appraisal of guidelines and practice. J Health Serv Res Policy. 2002;7(1):51-61. 